\title{
WT1 Positive
}

National Cancer Institute

\section{Source}

National Cancer Institute. WT1 Positive. NCI Thesaurus. Code C129453.

Indicates that WT 1 expression has been detected in a sample. 